\title{
Comparison of Cytologic Reports of Fine-Needle Aspiration of Thyroid Nodules with Pathologic Results of Thyroid Surgery
}

\author{
Akbar Jafari' ${ }^{1}$, Arash Dehghan², Farzaneh Esna-Ashari ${ }^{3}$, Shiva Borzouei,** \\ ${ }^{1}$ Internist, School of Medicine, Hamadan University of Medical Sciences, Hamadan, Iran \\ ${ }^{2}$ Assistant Professor, Department of Pathology, School of Medicine, Hamadan University of Medical Sciences, Hamadan, \\ Iran \\ ${ }^{3}$ Associate Professor, Department of Social Medicine, School of Medicine, Hamadan University of Medical Sciences, \\ Hamadan, Iran \\ ${ }^{4}$ Assistant Professor, Department of Endocrinology, School of Medicine, Hamadan University of Medical Sciences, Hamadan, \\ Iran \\ * Corresponding Author: Shiva Borzouei, Department of Endocrinology, School of Medicine, Hamadan University of \\ Medical Sciences, Hamadan, Iran. Email: borzooeishiva@yahoo.com
}

\begin{tabular}{|c|c|}
\hline & Abstract \\
\hline $\begin{array}{l}\text { Received: } 11.12 .2018 \\
\text { Accepted: } 13.04 .2019\end{array}$ & \multirow[b]{2}{*}{$\begin{array}{l}\text { Background and Objective: Thyroid nodules are among the most common } \\
\text { clinical problems and the application of an inexpensive and accurate } \\
\text { diagnostic method prevents performing inessential surgeries. In this regard, } \\
\text { the aim of this study was to compare the cytologic report of fine-needle } \\
\text { aspiration (FNA) of thyroid nodules with the results of thyroid surgery. } \\
\text { Materials and Methods: In this cross-sectional study, all the patients who } \\
\text { had referred to the clinic of Beheshti Hospital of Hamedan, Iran, due to } \\
\text { thyroid nodule in } 2017 \text { underwent FNA. The samples were then sent to the } \\
\text { pathology laboratory, and the cytology results were recorded in a checklist. } \\
\text { Based on the cytology results, a portion of these cases was referred to } \\
\text { undergo surgery. Following that, the sensitivity and specificity of cytology } \\
\text { results of thyroid nodules were calculated in comparison to pathology results } \\
\text { of the surgery sample. } \\
\text { Results: Out of } 115 \text { studied patients, } 18 \text { (15.65\%) and } 97 \text { ( } 84.35 \% \text { ) subjects } \\
\text { were male and female, respectively. The mean age of the subjects was } \\
40.14 \pm 12.34 \text { years. Most cases were in the form of the right-side unilateral } \\
\text { nodule ( } 46.1 \%) \text {. The number of benign and malignant cases diagnosed by } \\
\text { the pathologist was } 73 \text { (63.48\%) and } 42 \text { (36.52\%), respectively. The } \\
\text { specificity, sensitivity, as well as positive and negative predictive values } \\
\text { obtained from the FNA of thyroid nodules by the pathologist, were reported } \\
\text { as } 82.98 \% \text {, } 95.56 \%, 89.04 \text {, and } 92.68 \% \text {, respectively. It is necessary to } \\
\text { mention that the diagnostics accuracy of the pathologist associated with the } \\
\text { results of surgery pathology was } 90.43 \% \text {. } \\
\text { Conclusion: Performing FNA enjoys a relatively high specificity and } \\
\text { sensitivity as an inexpensive, low-risk, and simple method in the } \\
\text { differentiation of benign and malignant cases if it is performed precisely } \\
\text { from the site of the nodule with adequate biopsy and properly examined } \\
\text { pathologically. }\end{array}$} \\
\hline $\begin{array}{l}\text { How to Cite this Article: } \\
\text { Jafari A, Dehghan A, Esna-Ashari } \\
\text { F, Borzouei S. Comparison of } \\
\text { Cytologic Reports of Fine- } \\
\text { Needle Aspiration of Thyroid } \\
\text { Nodules with Pathologic Results } \\
\text { of Thyroid Surgery. Avicenna J } \\
\text { Clin Med. 2019; 26(1): } 20-25 \text {. } \\
\text { DOI: } 10.29252 / \text { ajcm.26.1.20 }\end{array}$ & \\
\hline & Keywords: Fine-Needle Aspiration, Thyroid Nodules, Thyroid Surgery \\
\hline
\end{tabular}


do): $10.29252 /$ ajcm.26.1.20

\title{
مقايسه تزارش سيتولوزى آسييراسيون سوزنى نازك ندولهاى تيروئيدى با نتايج ياتولوزى جراحى تيروئيد
}

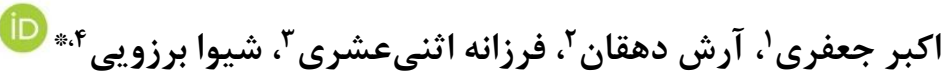 \\ ' ' متخصص داخلى، دانشكده يزشكى، دانشكاه علوم يزشكى همدان، همدان، ايران

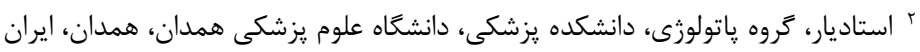

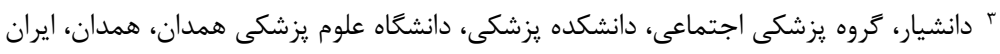

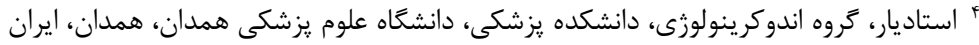
* نويسنده مسئول: شيوا برزويى، گروه اندوكرينولوزى، دانشكده يزشكى، دانشعاه علوم يزشكى همدان، همدان، ايران. ايميل: borzooeishiva@yahoo.com

\begin{tabular}{|c|c|}
\hline 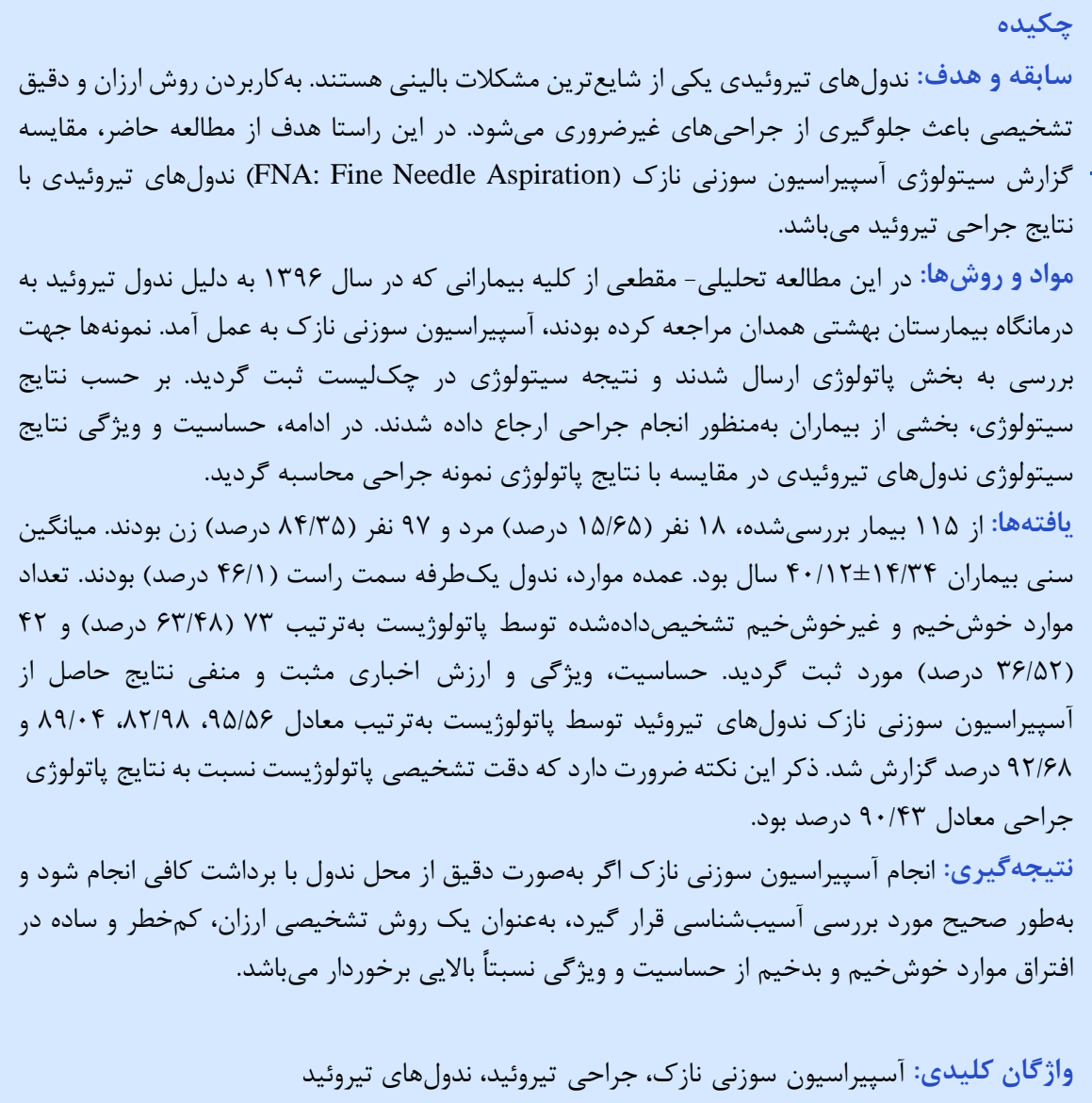 & 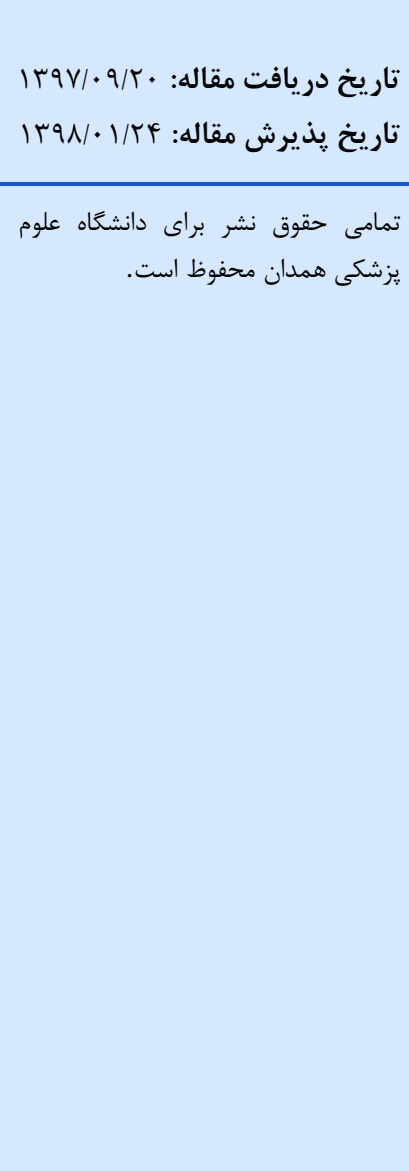 \\
\hline
\end{tabular}

كلوئيدى خوشخيم هسـتند [F] و كمتر از 1 تا 19 أدرصــ از

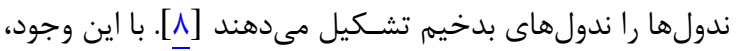

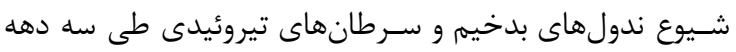

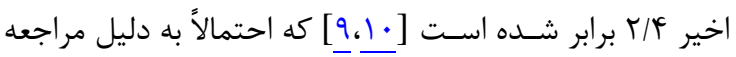

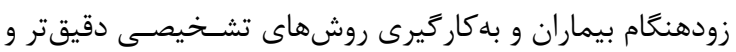

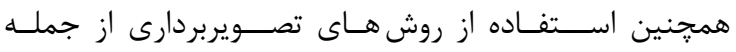

يكى از شــايع ترين دلايل مراجعه بيماران به كلينيك

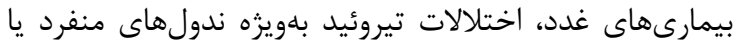

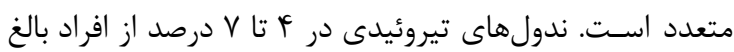

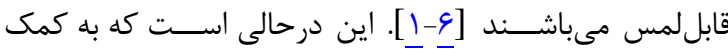

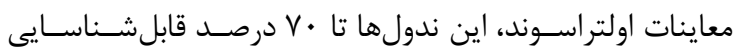

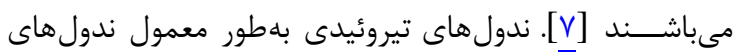


بيمـاران با تظاهرات بالينى ندول تيروئيدى منفرد يا متعدد

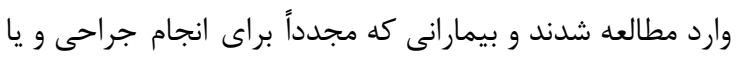
سـاير مراحل تشخيصى مراجعه ننمودند از مطالعه كنار كذاشته دئه

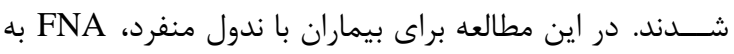

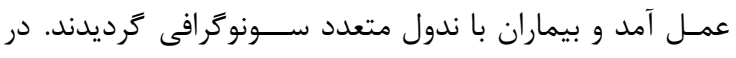

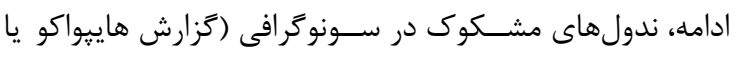

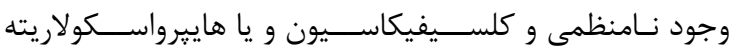
مركزى) تحت FNA قرار ترفتند (براى تمامى بيماران مورد

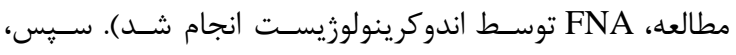

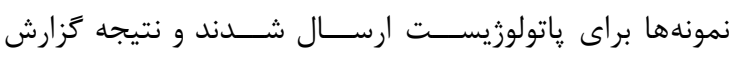

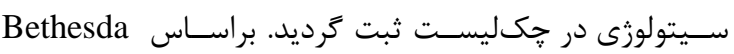

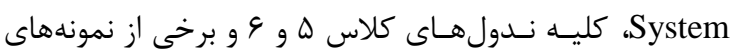

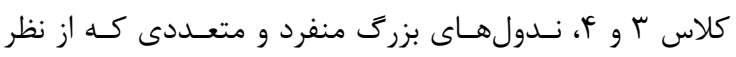

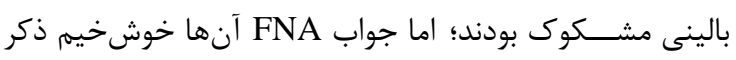
شـده بود و نيز ندولهايى كه علائم فشارى بر اركانهاى مجاب مجاور

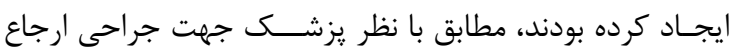

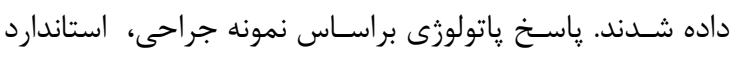

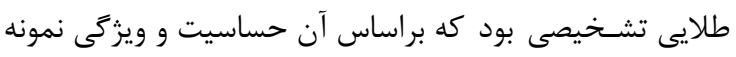
FNA

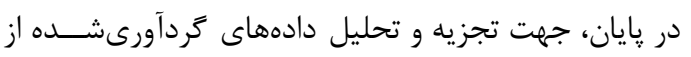

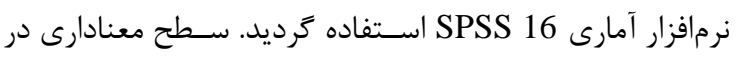

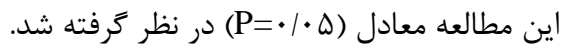

\section{يافته ها}

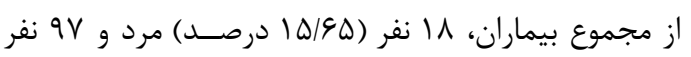

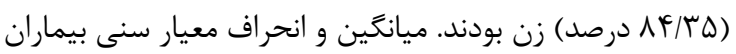

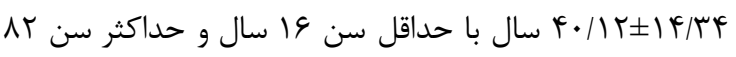

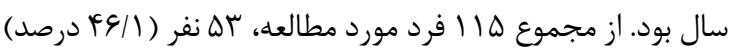

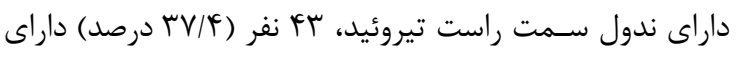

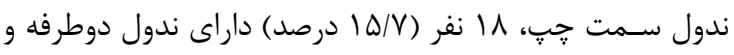

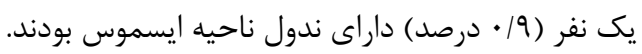

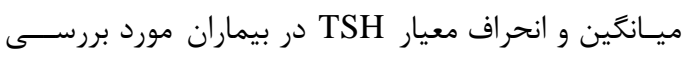

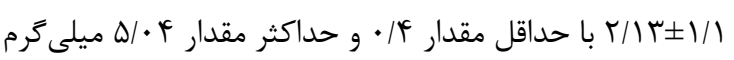

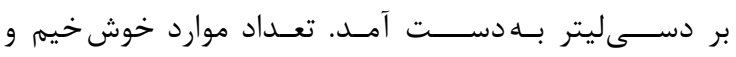
غيرخوش خيم تشخيصدادهشده توسط پاتولوزيست در جدول 1 كزارش شده است. تعداد موارد خوش خيم و بدخيم تشـخيص اسدادهده بر روى

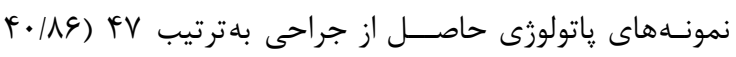

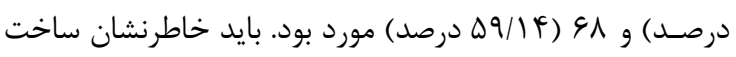

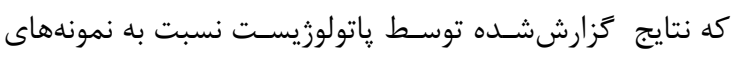

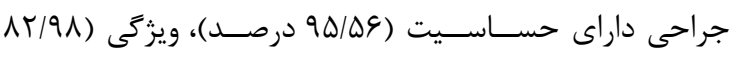

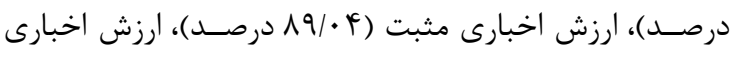

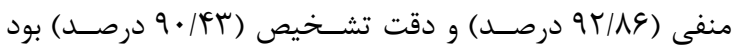

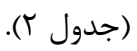

اولتراسونوگرافى، اسكن راديوايزوتوڤ، سى تى اسكن و ام آر اى

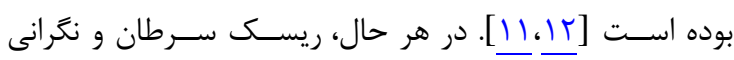
بيماران موجب نياز روزافزون به تشـيص و شــاسـايى صحيح

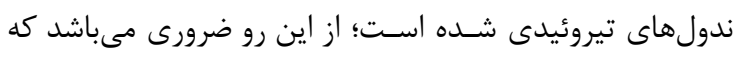

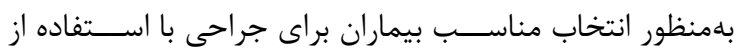
روشهاى مختلف، ندولهاى خوش خيم و بدخيم از يكديكر افتراق

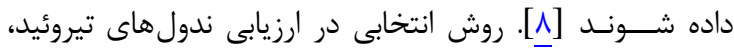

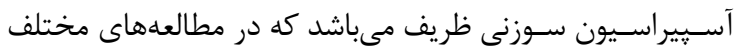

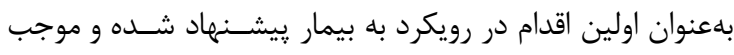

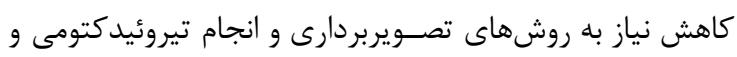

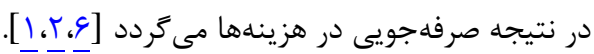

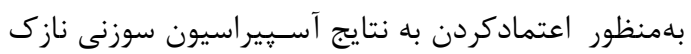

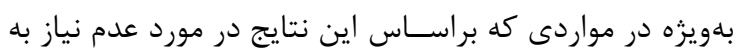

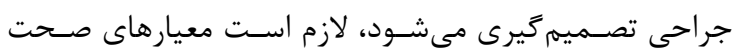

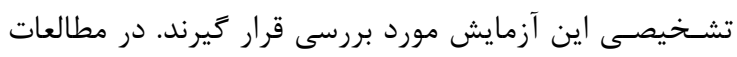

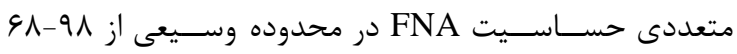

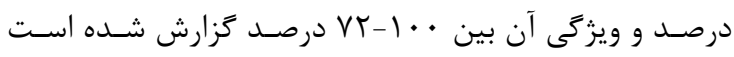

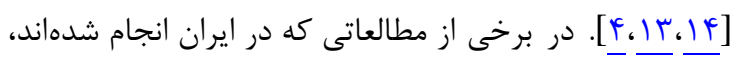

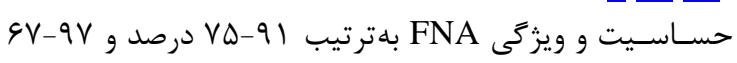

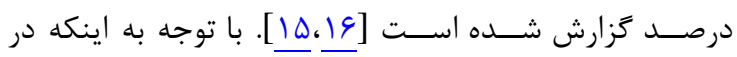
سال هاى اخير اين روش تشخيصى در كشور ما به فراوانى مورد

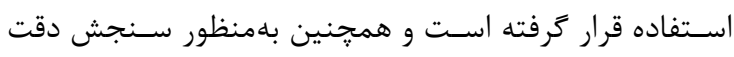

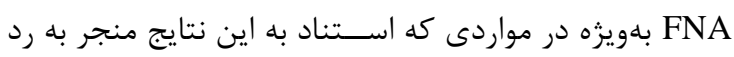

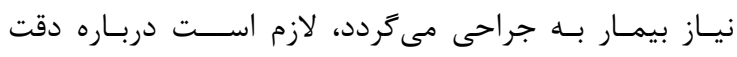

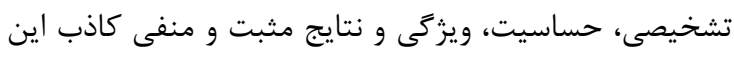

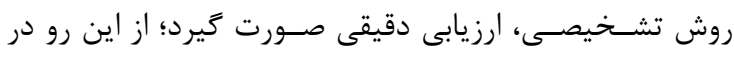
مطالعه حاضر ارزش تشخيصى FNA با نتايج جراحى تئى تيروئيد مورد مقايسه قرار زرفت.

\section{مواد و روشها}

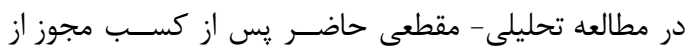

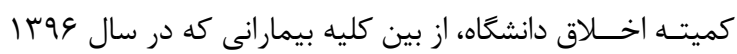

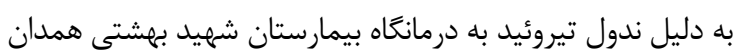

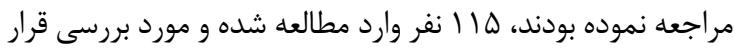

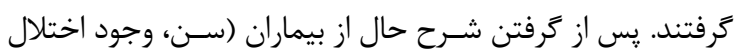

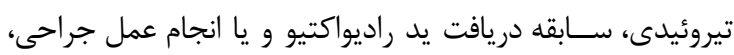

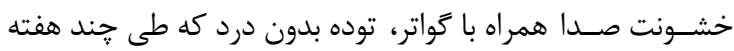

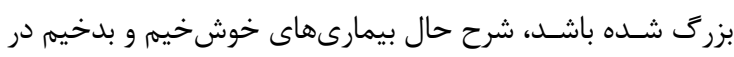

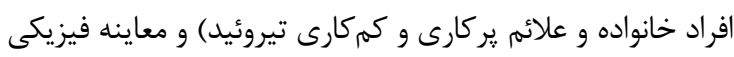

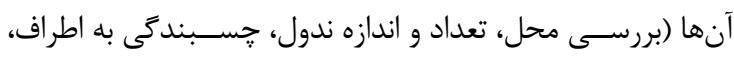

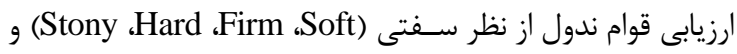

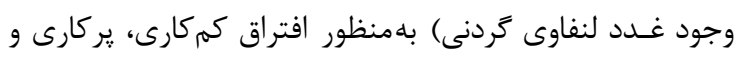

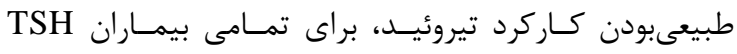
(Thyroid-stimulating Hormone) 
براساس آزمايش انجامشده بر روى نمونه ياتولوزى جراحى،

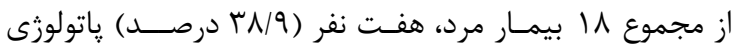

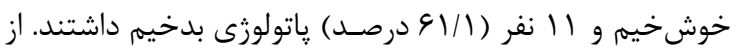

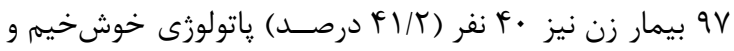

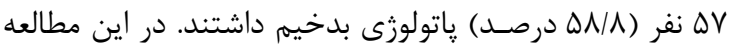

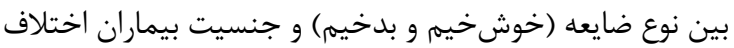

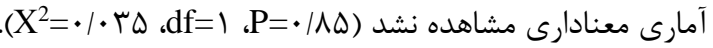

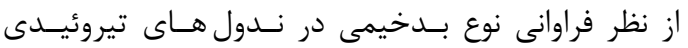

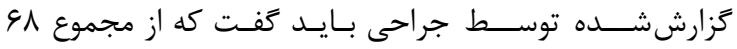

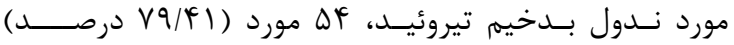

جدول ا: توزيع فراوانى انواع پاتولوزيك تشخيصدادهشده توسط

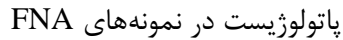

\begin{tabular}{|c|c|c|}
\hline \multicolumn{2}{|c|}{ تزارش هاتولوزيست } & \\
\hline درصد & تعداد & \\
\hline re/Q & FT & خوش خيم \\
\hline $11 / \pi$ & r & ضايعه آتيبيك \\
\hline $\mid N / T$ & rI & نئويلاسم فوليكولى \\
\hline$r q / 9$ & re & مشكوك به بدخيم \\
\hline$F / T$ & $\Delta$ & بدخيم \\
\hline $1 \cdots$ & 110 & جمع \\
\hline
\end{tabular}

جدول Y: مقايسه نتايج سيتولوزى آسيراسيون سوزنى نازى در ندولهاى تيروئيدى گزارششده توسط پاتولوزيست با نتيجه ياتولوزى جراحى

\begin{tabular}{|c|c|c|c|c|}
\hline جمع & بدخيم & خوش خيجه ياتولو & & \\
\hline ET & r & rq & خوش خيم & \multirow{3}{*}{ كزارش سيتولوزى } \\
\hline Vr & $9 \Delta$ & $\wedge$ & بدخيم & \\
\hline 110 & 91 & FV & جمع & \\
\hline
\end{tabular}

آمارى كه در كشـور آمريكا منتشر شده است، حدود بأ درصد از

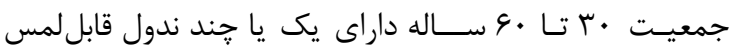
هسـتند و ميزان وجود ندول تيروئيد در زنان، جهار برابر مردان

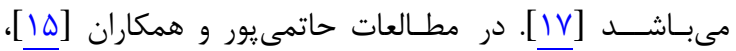

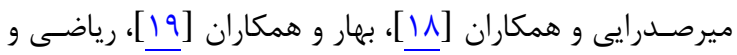

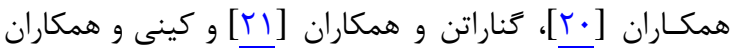

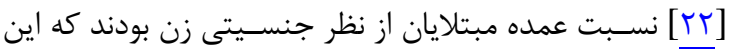

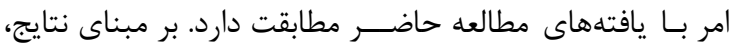

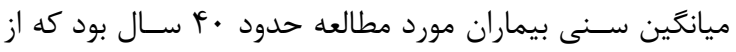

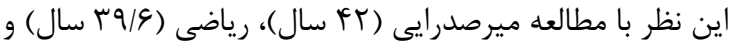

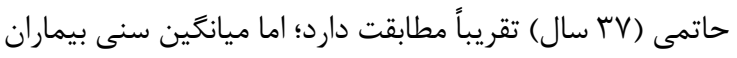

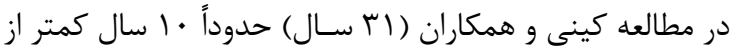
يافتههاى مطالعه حاضر بود. به نظر مىرسد كه اين امر مى متواند ناشى از توزيع نزادى و جغرافيايى منطقه مربوطه باشد.

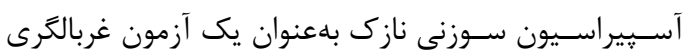

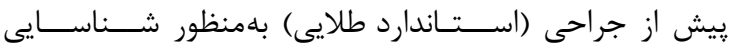

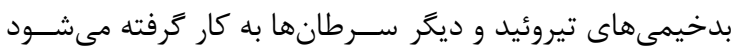

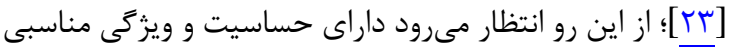

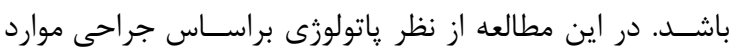

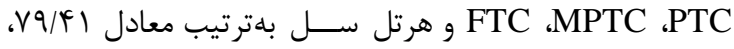

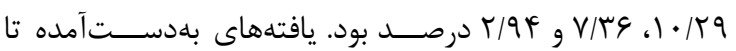

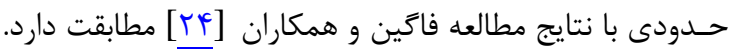

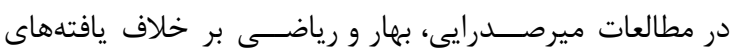
مطالعه حاضـر، نسـبت موارد خوش خيم بيشـتر از موارد بدخيم

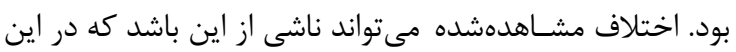

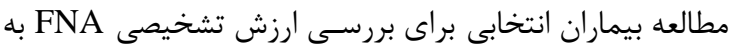

Papillary Thyroid Carcinoma Micro Papillary Thyroid Carcinoma درصد) Follicular Thyroid Cancer و دو مورد (F/9Y م/ درصد)

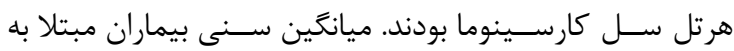

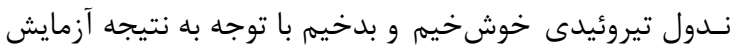

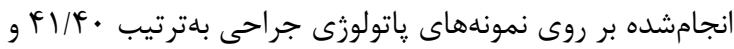

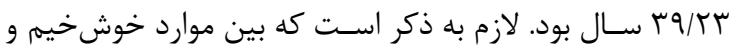
بدخيم تشـخيصدادهشده از نظر سنى اختلاف آمارى معنادارى درى

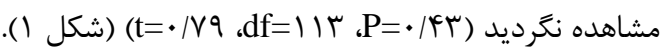

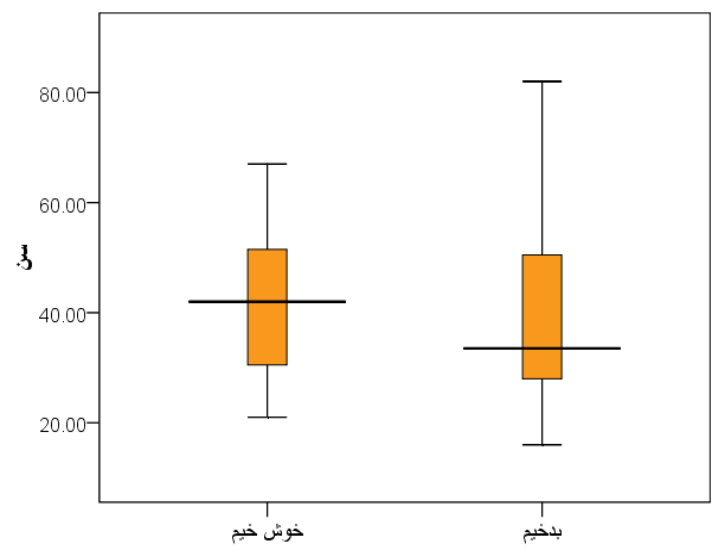

شكل ا: ميانه سنى بيماران مبتلا به ندولهاى تيروئيدى بر حسب

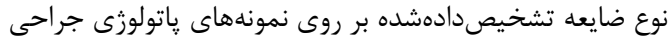

بحث

ندولهاى تيروئيدى يكى از شـايعترين شــايتهاى افراد

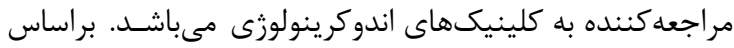




$$
\text { مى يابد [r] [r. }
$$

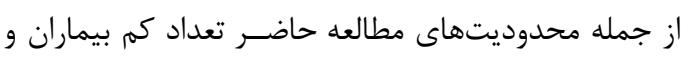

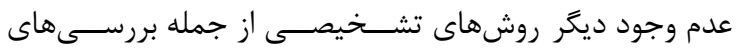
مولكولى و آزمونهاى سـيتولوزى دريكر روشئ محيط مايع بود. در صورت دسترسى به اين شيوههاى تشخيصى مى متوان مطالعات جديدى

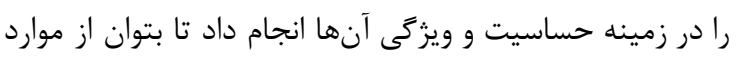
ارجاع غيرضرورى جهت انجام جراحى كاست.

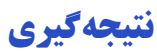

انجام آسييراسيون سوزنى نازى اگر بلهورت دقيق از محل

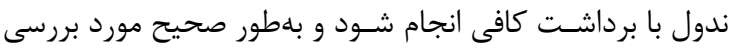

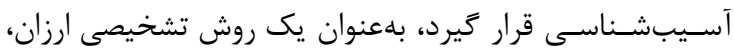

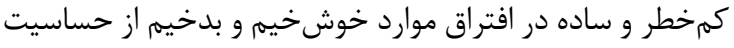

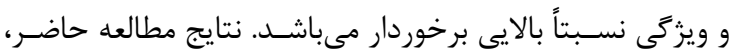

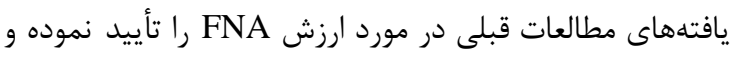
به دانشافزايى در اين مورد كمك مى كند.

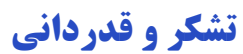

مقاله حاضر بركرفته از ياياننامه دوره دستيارى طب داخلى

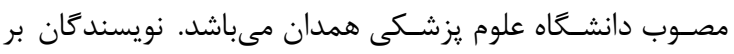

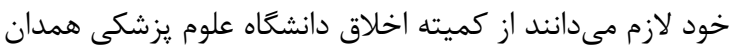

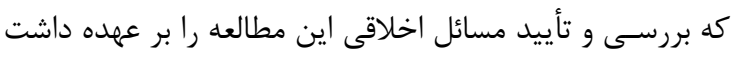

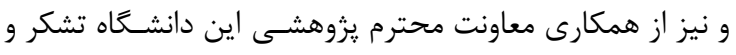

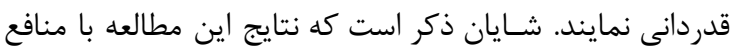
نويسندكان در تعارض نمى باشد.

\section{REFERENCES}

1. Gharib H, Goellner JR. Fine-needle aspiration biopsy of the thyroid: an appraisal. Ann Intern Med. 1993;118(4):282-9. PMID: 8420446

2. Hegedüs L. Clinical practice. The thyroid nodule. $N$ Eng J Med. 2004;351(17):1764-71. PMID: 15496625 DOI: 10.1056/NEJMcp031436

3. Lew JI, Snyder RA, Sanchez YM, Solorzano CC. Fine needle aspiration of the thyroid: correlation with final histopathology in a surgical series of 797 patients. $J$ Am Coll Surg. 2011;213(1):188-94. PMID: 21601489 DOI: 10.1016/j.jamcollsurg.2011.04.029

4. Ogilvie JB, Piatigorsky EJ, Clark OH. Current status of fine needle aspiration for thyroid nodules. Adv Surg. 2006;40:223-38. PMID: 17163105

5. Porterfield JR Jr, Grant CS, Dean DS, Thompson GB, Farley DR, Richards ML, et al. Reliability of benign fine needle aspiration cytology of large thyroid nodules. Surgery. 2008;144(6):963-8. PMID: 19041004 DOI: 10.1016/j. surg.2008.09.006

6. Sclabas GM, Staerkel GA, Shapiro SE, Fornage BD, Sherman SI, Vassillopoulou-Sellin R, et al. Fine-needle aspiration of the thyroid and correlation with histopathology in a contemporary series of 240 patients. Am J Surg. 2003;186(6):702-9. PMID: 14672783

7. Goldfarb M, Gondek S, Solorzano C, Lew JI. Surgeonperformed ultrasound can predict benignity in thyroid nodules. Surgery. 2011;150(3):436-41. PMID: 21878228 DOI: 10.1016/j.surg.2011.07.002

8. Burman KD, Wartofsky L. Thyroid nodules. $N$ Engl J Med.
لحاظ حســاســيت و ويزگگى مواردى بودند كه داراى نتيجه

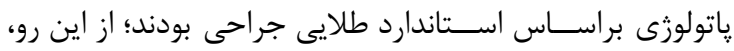

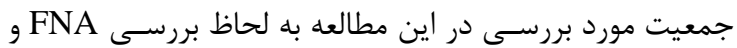

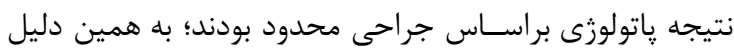

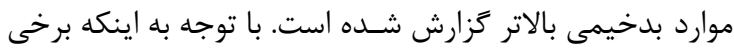

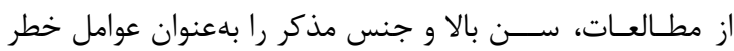

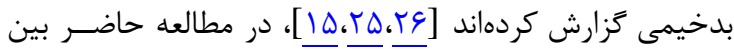

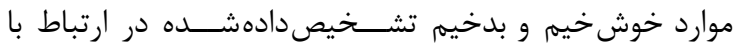

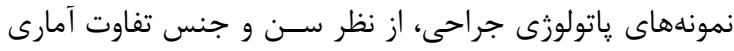

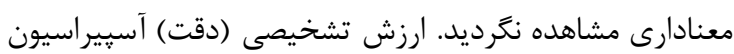

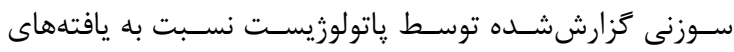

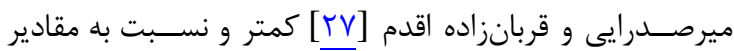

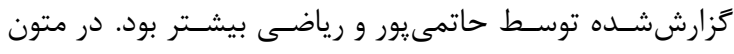

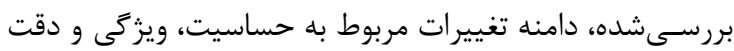

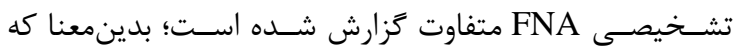

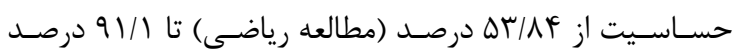

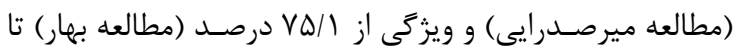

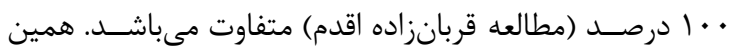

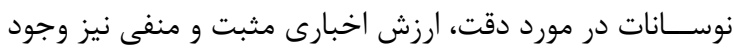

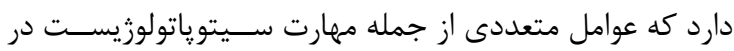

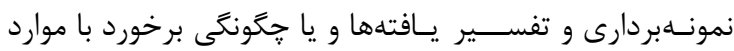
مشكوك به بدخيمى در يافتههاى FNA در اين تغييرات دخيل

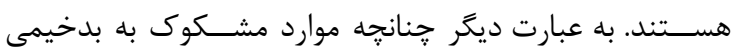

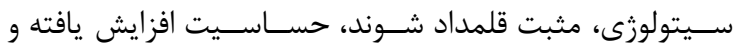

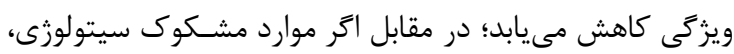

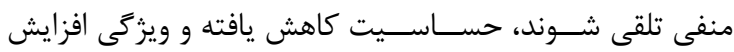

2016;374(13):1294-5. PMID: 27028927 DOI: 10.1056/ NEJMc1600493

9. Cooper DS, Doherty GM, Haugen BR, Kloos RT, Lee SL, Mandel SJ, et al. Revised American Thyroid Association management guidelines for patients with thyroid nodules and differentiated thyroid cancer. Thyroid. 2009;19(11):1167214. PMID: 19860577 DOI: $10.1089 /$ thy.2009.0110

10. Yassa L, Cibas ES, Benson CB, Frates MC, Doubilet PM, Gawande AA, et al. Long-term assessment of a multidisciplinary approach to thyroid nodule diagnostic evaluation. Cancer. 2007;111(6):508-16. PMID: 17999413 DOI: $10.1002 /$ cncr.23116

11. Roy R, Kouniavsky G, Venkat R, Felger EA, Shiue Z, Schneider E, et al. The role of preoperative neck ultrasounds to assess lymph nodes in patients with suspicious or indeterminate thyroid nodules. J Surg Oncol. 2012;105(6):601-5. PMID: 22006435 DOI: $10.1002 /$ jso.22115

12. Wang C, Friedman L, Kennedy GC, Wang H, Kebebew E, Steward DL, et al. A large multicenter correlation study of thyroid nodule cytopathology and histopathology. Thyroid. 2011;21(3):243-51. PMID: 21190442 DOI: 10.1089/thy. 2010.0243

13. Al-azawi D, Mann GB, Judson RT, Miller JA. Endocrine surgeon-performed US guided thyroid FNAC is accurate and efficient. World J Surg. 2012;36(8):1947-52. PMID: 22526037 DOI: $10.1007 / \mathrm{s} 00268-012-1592-2$

14. Pinchot SN, Al-Wagih H, Schaefer S, Sippel R, Chen H. Accuracy of fine-needle aspiration biopsy for predicting neoplasm or carcinoma in thyroid nodules $4 \mathrm{~cm}$ or larger. 
Arch Surg. 2009;144(7):649-55. PMID: 19620545 DOI: 10.1001/archsurg.2009.116

15. Hatamipoor E, Khosravi A, Amjadimanesh J, Fatthee P. A study on diagnostic value of fine needle aspiration (fna) test in determination of malignant thyroid nodules based on pathologic findings. Armaghane Danesh. 2005;10(37):53-8. [Persian]

16. Sirous M, Jianpour M, Rezaei A, Saadatpour Z. Comparison the color-doppler ultra sonography thyroid Nodules Criteria and FNA Findings. J Isfahan Med Sch. 2011;28(120):1-7. [Persian]

17. Kasper D, Fauci A, Hauser S. Harrison's principles of internal medicine. New York: McGraw-Hill; 2005.

18. Mirsadraee S, Mousavi Z, Farzadnia M, Bavafa A, Kakhi S. Evaluation of diagnostic value of fine needle aspiration in thyroid nodules. Med J Mashhad Univ Med Sci. 2007;50(1):23-30. [Persian]

19. Bahar A, Zkashi Z, Akha O. The result of fine-needleaspiration of thyroid nodule in patient referred to Imam Hospital Sari 2003-2011. J Mazandaran Univ Med Sci. 2012;22(90):11-6. [Persian]

20. Riazi A, Eghbali SS, Bahmanyar M, Farzaneh M, Motlagh FR, Motamed N, et al. Correlation of fine needle aspiration of the thyroid with final histopathology in 198 thyroidectomized patients. Iran South Med J. 2013;16(1):3748. [Persian]

21. Gunaratne SA, Wijesinghe HK, Wijesinghe CJ, Dissanayake SK, Godakandage MH. Comparison of Fine Needle
Aspiration Cytology (FNAC) and thyroid ultrasonography in the diagnosis of thyroid nodules. J Diagn Pathol. 2016;10(2):12-20.

22. Kini U, Buch A, Bantwal G. Role of FNA in the medical management of minimally enlarged thyroid. Diagn Cytopathol. 2006;34(3):196-200. PMID: 16470863 DOI: $10.1002 / \mathrm{dc} .20415$

23. Theoharis CG, Schofield KM, Hammers L, Udelsman R, Chhieng DC. The Bethesda thyroid fine-needle aspiration classification system: year 1 at an academic institution. Thyroid. 2009;19(11):1215-23. PMID: 19888859 DOI: 10.1089/thy.2009.0155

24. Fagin JA, Wells SA Jr. Biologic and clinical perspectives on thyroid cancer. $N$ Engl J Med. 2016;375(23):2307. PMID: 27959677 DOI: 10.1056/NEJMc1613118

25. Ramzy I. Clinical cytopathology and aspiration biopsy: fundamental principles and practice: New York: McGraw Hill; 2001.

26. Rosai J. Special techniques in surgical pathology. In: Rosai and Ackerman's surgical pathology. Philadelphia: Mosby, 2004. P. 37-91.

27. Ghorbanzdeh Aghdam N. Evaluation the diagnostic value of fine needle aspiration biopsy done in Loghman hospital, Tehran as an inexpensive method of diagnosing malignancy as compared with histopathology reports as the standard test. [Doctor Dissertation]. Tehran: School of Medicine, Shaheed Beheshti University of Medical Sciences; 2013. 